\title{
Farinha da casca do fruto de Passiflora edulis f. flavicarpa Deg (maracujá-amarelo): do potencial terapêutico aos efeitos adversos
}

\author{
COQUEIRO, A.Y. ${ }^{1 *}$; PEREIRA, J.R.R. ${ }^{2}$; GALANTE, F. ${ }^{2}$
}

Universidade de São Paulo - Faculdade de Ciências Farmacêuticas, Avenida Professor Lineu Prestes, $n^{\circ} 580$, CEP: 05508-000, São Paulo - SP, Brasil. 2Faculdades Metropolitanas Unidas (FMU). Rua Taguá, $n^{\circ} 150$, CEP: 01508-010, São Paulo - SP, Brasil. *Autor para correspondência: audreycoqueiro@hotmail.com

\begin{abstract}
RESUMO: O maracujá-amarelo (Passiflora edulis f. flavicarpa Deg) é considerado um alimento funcional, visto que, além de funções nutricionais, desempenha ações em vias metabólicas específicas. A farinha da casca do maracujá-amarelo (Passiflora edulis f. flavicarpa Deg) é rica em pectina. Esta fibra reduz a absorção de glicídios e lipídios, influenciando no metabolismo destes nutrientes. Portadores de patologias associadas às alterações no metabolismo glicêmico e lipidêmico têm utilizado este recurso de forma indiscriminada. Porém, substâncias designadas glicosídeos cianogênicos, presentes na casca do fruto são tóxicas ao organismo e prejudiciais à saúde. Ademais, o uso exacerbado de agrotóxicos na produção do maracujá é preocupante e a ingestão destes compostos também pode acarretar complicações à saúde. Portanto, o objetivo do presente trabalho foi sintetizar o conhecimento disponível referente aos efeitos terapêuticos e colaterais da suplementação com a farinha da casca do maracujá-amarelo (Passiflora edulis $\mathrm{f}$. flavicarpa Deg). Foi realizada uma revisão bibliográfica nas bases de dados indexadas SCIELO, LILACS e PUBMED, sem limitação quanto ao período de publicação. Os estudos inclusos no trabalho, de forma unânime, apresentam eficácia da suplementação com a farinha da casca do maracujá-amarelo. A trituração da casca do fruto, realizada no processo de obtenção da farinha, é incapaz de promover redução de glicosídeos cianogênicos e agrotóxicos, permitindo que os indivíduos submetidos à suplementação estejam susceptíveis aos efeitos tóxicos destes compostos. Dessa forma, o desenvolvimento de estudos que avaliem a toxicidade da suplementação por períodos crônicos é de suma importância. Referente ao uso de agrotóxicos, a produção da farinha com frutos orgânicos pode ser uma alternativa para atenuar a toxicidade, sendo necessária a elucidação desta hipótese através de estudos.
\end{abstract}

Palavras-chave: Passiflora, Maracujá, Agrotóxicos, Cianeto.

\begin{abstract}
Peel flour of the Passiflora edulis f. flavicarpa Deg (yellow passion fruit): From therapeutic potentials to side effects. The yellow passion fruit (Passiflora edulis $\mathrm{f}$. flavicarpa Deg) is considered a functional food, because, in addition to nutritional functions, it performs actions in specific metabolic pathways. The yellow passion fruit peel flour (Passiflora edulis f. flavicarpa Deg) is rich in pectin. This fiber reduces the absorption of carbohydrates and lipids, influencing the metabolism of these nutrients. Individuals affected by pathologies associated with alterations in the metabolism of lipids and carbohydrates have used this resource indiscriminately, however, substances called cyanogenic glycosides, present in the fruit peel, are toxic to the body and harmful to health. In addition, the overuse of pesticides in passion fruit production is becoming worrisome and the ingestion of these compounds can also cause negative health implications. Therefore, the aim of this study was to synthesize the available knowledge regarding the therapeutic effects and side effects of supplementation with the yellow passion fruit peel flour (Passiflora edulis f. flavicarpa Deg). A literature review was conducted from the indexed databases SCIELO, LILACS and PUBMED, without limitation to the publication period. The studies included in the work, unanimously, have effectiveness of supplementation with yellow passion fruit peel flour (Passiflora edulis f. flavicarpa Deg). The crushing of the fruit peel, performed in the process of obtaining flour, is unable to promote a reduction of cyanogenic glycosides and pesticides, causing individuals undergoing supplementation to be susceptible to the toxic effects of these compounds. Thus, this study's development to evaluate the toxicity of
\end{abstract}

Recebido para publicação em - 01/10/2015

Aceito para publicação em - 06/05/2016

10.1590/1983-084X/15_187

Rev. Bras. PI. Med., Campinas, v.18, n.2, p.563-569, 2016. 
supplementation for chronic periods is very important. With reference to the use of pesticides, flour production with organic fruits can be an alternative to attenuate toxicity and it is necessary to elucidate this hypothesis through this study.

Key words: Passiflora, Passion Fruit, Pesticides, Cyanide.

\section{INTRODUÇÃO}

A espécie Passiflora edulis, pertencente ao gênero Passiflora L, é, vastamente, conhecida por suas propriedades terapêuticas (Bellon et al., 2007). De forma semelhante, o fruto desta espécie, maracujá-amarelo (Passiflora edulis f. flavicarpa Deg), tem sido apontado como um alimento funcional por atuar em respostas fisiológicas específicas (Zeraik et al., 2010).

A farinha da casca do maracujá-amarelo (Passiflora edulis f. flavicarpa Deg) é obtida a partir da trituração da casca do fruto, a qual apresenta quantidades elevadas da fibra pectina. Esta fibra promove aumento de volume do bolo alimentar e da viscosidade das soluções no trato gastrointestinal, promovendo saciedade. O retardo no esvaziamento gástrico promovido pela pectina reduz o pico glicêmico decorrente da ingestão elevada de carboidrato (Souza et al., 2008), reduzindo a absorção de glicídios. Ademais, a pectina favorece a formação de uma camada gelatinosa na mucosa intestinal, reduzindo a absorção de lipídeos (Medeiros et al., 2009). Desta forma, este recurso tem sido empregado em estados patológicos caracterizados por alterações no metabolismo glicêmico e lipidêmico.

As folhas e o fruto imaturo do maracujáamarelo (Passiflora edulis f. flavicarpa Deg) possuem glicosídeos cianogênicos (Medeiros et al., 2009), como a prunasina, sambunigrina e amigdalina (Matsuura et al., 2005). A ingestão destes compostos é tóxica e, como resultado, culmina em distúrbios respiratórios (Medeiros et al., 2009), vertigem, náuseas, vômitos, diarreia e fraqueza (Do Nascimento et al., 2013). Além disso, o uso indiscriminado de agrotóxicos na produção do maracujá, a fim de combater doenças e pragas, pode repercutir em implicações à saúde (Faleiro et al., 2005).

Em virtude dos possíveis efeitos terapêuticos e colaterais da farinha da casca do maracujá-amarelo (Passiflora edulis f. flavicarpa Deg), o presente trabalho teve a perspectiva de investigar o emprego da suplementação com a farinha, a aplicabilidade deste recurso em eventos patológicos, bem como apontar meios capazes de atenuar os efeitos deletérios desta intervenção. Desta forma, o objetivo do trabalho foi sintetizar o conhecimento disponível a respeito dos efeitos terapêuticos e adversos da suplementação com a farinha da casca do fruto maracujá-amarelo (Passiflora edulis f. flavicarpa Deg).

\section{METODOLOGIA}

Foi realizada uma busca nas bases de dados indexadas SCIELO, LILACS e PUBMED. Os descritores utilizados para pesquisa foram extraídos do DeCS (Descritores em Ciências da Saúde) e $\mathrm{MeSH}$ (Medical Subject Headings), nos idiomas Inglês e Português. Foram utilizados os seguintes termos: "Passiflora", "Maracujá", "Agrotóxicos", "Cianeto". Não houve limitação quanto ao período de publicação, sendo incluídos trabalhos de 1988 a 2015. Os estudos experimentais encontrados, envolvendo a administração com a farinha da casca do maracujá-amarelo, foram publicados entre os anos 2002 e 2012. Foram incluídos artigos de revisão e artigos originais com humanos e animais, saudáveis ou em estado patológico, em diferentes ciclos da vida, envolvendo suplementação com a farinha da casca do maracujá-amarelo (Passiflora edulis f. flavicarpa Deg).

Passiflora edulis: origem, potencial terapêutico e aplicabilidade em processos patológicos

A espécie Passiflora edulis é a mais cultivada do gênero Passiflora $L$. Este é considerado o mais importante da família Passifloraceae, no que concerne ao aspecto econômico (Bellon et al., 2007). É representado por cerca de 450 espécies, aproximadamente 200 delas nativas do Brasil, onde são, popularmente, conhecidas como maracujás (Souza et al, 2008).

As principais ações terapêuticas da $P$. edulis são: atividade depressiva do sistema nervoso central, efeito ansiolítico, sedativo, anticonvulsivante (Vargas et al., 2007), potencial antioxidante (Souza et al., 2012; Konta et al., 2014), anti-inflamatório (Montanher et al., 2007; Benincá et al., 2007), tratamento da hipertensão (Konta et al., 2014), ações cicatrizantes em lesões cutâneas, feridas, erisipelas, entre outras (Bezerra et al., 2006).

Deng et al. (2010) constataram ação da $P$. edulis no sistema nervoso após submeter camundongos ao tratamento com extrato etanólico da planta. Em doses reduzidas houve efeito ansiolítico da intervenção, entretanto, doses 
elevadas resultaram em efeito sedativo.

Referente aos efeitos antioxidantes, Souza et al. (2012) observaram atenuação de parâmetros de estresse oxidativo (peroxidação lipídica) em ratos submetidos ao tratamento com o suco do fruto da Passiflora edulis f. flavicarpa Deg (maracujá-amarelo). De forma semelhante, Konta et al. (2014) administraram a polpa do fruto da $P$. edulis em ratos hipertensos e como resultados foi observado redução de parâmetros de estresse oxidativo, elevação nos níveis de glutationa reduzida (antioxidante) e redução significativa na pressão sanguínea sistólica.

Bezerra et al. (2006) avaliaram em ratos submetidos a cirurgia de secção da alça colônica, o efeito de um extrato etanólico elaborado a partir das folhas de $P$. edulis. Neste trabalho o grupo tratado apresentou cicatrização otimizada, quando comparado ao grupo placebo. Ademais, a intervenção atenuou o processo inflamatório subagudo. Vagas et al. (2007) administraram extrato aquoso de $P$. edulis em camundongos e observaram redução de parâmetros inflamatórios, conferindo a este fitoterápico atividade anti-inflamatória.

Além destas ações, Gonçalves Filho et al. (2006) observaram que o extrato hidroalcoólico das folhas de $P$. edulis elevou a proliferação fibroblástica, colagenização e neoformação capilar na bexiga de ratos. De forma similar, Garros et al. (2006) observaram elevação, a nível microscópico, da proliferação fibroblástica e colagenização em tecidos lesionados de ratos, após administração com extrato hidroalcoólico das folhas de $P$. edulis.

Estas propriedades terapêuticas são provenientes da ação fisiológica de substâncias químicas existentes nesta espécie, dentre elas, alcaloides (harmol, harmana, harmina e harmalina), carotenoides e flavonoides (Bezerra et al., 2006).

Estudos têm avaliado, não somente as ações terapêuticas das folhas, mas também do fruto da $P$. edulis (Ramos et al., 2007; Janebro et al., 2008; Medeiros et al., 2009). A farinha da casca do maracujá-amarelo (Passiflora edulis f. flavicarpa Deg) é categorizada como um alimento funcional por apresentar, além de funções nutricionais, ações em respostas fisiológicas específicas, as quais podem prevenir ou auxiliar na recuperação de doenças (Zeraik et al., 2010).

\section{Maracujá-amarelo (Passiflora edulis f. flavicarpa Deg): composição nutricional e potencial terapêutico \\ O maracujá-amarelo (Passiflora edulis $\mathrm{f}$.} flavicarpa Deg), também denominado "maracujáazedo", é o fruto mais explorado (Marchi et al., 2000) e relevante na produção de sucos (Freitas \& Oliveira Filho, 2003), comparado às demais espécies de Passiflora nativas do Brasil (Marchi et al., 2000). Cerca de $52 \%$ da composição mássica do fruto é constituída pela casca (Ishimoto et al., 2007). A porção branca da mesma é rica em niacina, ferro (Oliveira et al., 2002; Córdova et al., 2005), cálcio e fósforo (Ferrari et al., 2004). O teor de fibra bruta da casca é superior ao da polpa, especialmente, em pectinas e mucilagens (Córdova et al., 2005).

O termo "fibra alimentar" designa a associação de diferentes tipos de polissacarídeos de origem vegetal, os quais são resistentes à hidrólise, ou seja, ação das enzimas do trato digestório (Ramos et al., 2007). Elevados teores da fibra pectina estão associados ao aumento de volume do bolo alimentar e da viscosidade das soluções no trato gastrointestinal, promovendo saciedade. $O$ retardo no esvaziamento gástrico, promovido pela fibra, reduz o pico glicêmico decorrente da ingesta de elevadas quantidades de carboidrato (Souza et al., 2008), através da formação de uma camada gelatinosa na mucosa intestinal, a qual impede a absorção de glicídios e lipídios (Medeiros et al., 2009).

No intestino grosso, a pectina, após ser fermentada, origina ácidos graxos de cadeia curta (acetato, butirato e propianato). Estudos indicam que o butirato está associado à redução da resistência à insulina nos tecidos periféricos, enquanto o propianato, através de seu metabolismo hepático, estimula à gliconeogênese e lipogênese. Estas ações conferem à pectina propriedades anticancerígenas e imunoestimulatórias (Braga et al., 2010).

O somatório das propriedades descritas fundamenta o emprego do uso da farinha da casca do fruto maracujá-amarelo (Passiflora edulis f. flavicarpa Deg) em estados patológicos caracterizados por alterações no metabolismo glicêmico e lipidêmico, bem como a fim de prevenir doenças.

\section{Estudos experimentais}

Os estudos destinados a avaliar os efeitos da farinha da casca do fruto maracujá-amarelo (Passiflora edulis f. flavicarpa Deg) tornaram-se mais frequentes a partir dos anos 2000. Guertzenstein \& Sabaa-Srur (2002) estudaram a administração com a farinha na ração de ratos diabéticos e nãodiabéticos. Os ratos não-diabéticos e tratados consumiram menos ração e apresentaram menor ganho de peso, comparado ao grupo não-diabético placebo. Ambos os grupos submetidos a intervenção apresentaram redução na glicemia.

Em 2010, Braga et al. observaram redução da glicemia em ratos diabéticos duas horas após administração com a farinha. Segundo os autores, a ação é decorrente da atividade da pectina no

Rev. Bras. PI. Med., Campinas, v.18, n.2, p.563-569, 2016. 
intestino delgado, reduzindo a absorção de glicose, em sinergia a atuação da fibra no intestino grosso, promovendo síntese de ácidos graxos de cadeia curta.

Os resultados promissores obtidos em estudos experimentais com animais impulsionaram o desenvolvimento de estudos aplicados em humanos. No ano de 2007, Ramos et al. submeteram 19 pacientes a suplementação com a farinha. Após oito semanas de intervenção, houve redução do colesterol total e fração LDL nos grupos tratados, mas os valores de HDL-colesterol mantiveramse estáveis. Não foram observadas alterações de parâmetros hepáticos, tais como bilirrubina total, direta, indireta e gama GT (GGT), indicando ausência de toxicidade nos grupos tratados.

Janebro et al., (2008) observaram ação hipoglicemiante no tratamento com a farinha em portadores de diabetes mellitus tipo II. Observouse redução nos valores de glicemia em jejum, hemoglobina glicada $(\mathrm{HbA1c})$ e triglicérides após a intervenção, bem como aumento na fração HDLcolesterol.

No intuito de avaliar, não somente o efeito terapêutico, mas também o potencial tóxico da intervenção, Medeiros et al. (2009) submeteram indivíduos a administração com a farinha durante oito semanas. O tratamento promoveu redução significativa na glicemia e peso corporal $(2 \%$ de redução) dos indivíduos, comparado aos valores pré-intervenção. Não foi observada toxicidade ou efeitos colaterais nesta dosagem e período experimental.

Queiroz et al. (2012) administraram a farinha em portadores de diabetes mellitus tipo II e constataram redução de $14,6 \%$ nos níveis de glicose plasmática durante os primeiros 30 dias de experimento e, $25,7 \%$ após 60 dias. Os valores de hemoglobina glicada reduziram significativamente após intervenção.

Embora os estudos apresentem resultados promissores, em decorrência ao forte sabor residual e distúrbios gastrointestinais provocados pelo uso da farinha, alguns voluntários tendem a interromper sua participação nas pesquisas (Ramos et al., 2007). Substâncias designadas glicosídeos cianogênicos são tóxicas e apresentam-se em quantidades significativas em todas as partes da Passiflora edulis (Medeiros et al., 2009). Além disso, o uso de agrotóxicos de forma indiscriminada na produção do maracujá-amarelo apresenta riscos à saúde (Faleiro et al., 2005).

Na Figura 1 são apresentados os estudos supracitados e seus respectivos protocolos experimentais.

\section{Glicosídeos cianogênicos: potencial tóxico e implicações à saúde \\ O ácido cianídrico é um líquido volátil e} incolor, considerado uma das substâncias mais tóxicas existentes. Normalmente está associado a um aldeído ou uma cetona e a um ou mais açucares, formando compostos denominados glicosídeos cianogênicos. Atualmente há, aproximadamente, 120 plantas que contém estes compostos em quantidade suficiente para provocar intoxicação, que ocorre após ingestão de quantidades elevadas em curtos períodos de tempo (Carvalho et al., 2011).

Embora seja considerado um sistema próprio de defesa das plantas, quando ocorre rompimento do tecido vegetal, há um desdobramento de reações enzimáticas entre os glicosídeos até o ácido cianídrico. Na corrente sanguínea, este ácido libera íon cianeto, o qual se liga ao citocromo mitocondrial da hemoglobina. Esse processo é tóxico e acarreta em asfixia celular que, dependendo do teor de cianeto no sangue, pode levar à morte (Do Nascimento et al., 2013).

No intuito de reduzir o teor destes compostos, Matsuura et al. (2005) avaliaram o efeito da trituração do fruto maracujá-amarelo (Passiflora edulis f. flavicarpa Deg) associado a imersão em água. O processo de imersão provocou redução de parte dos glicosídeos cianogênicos, entretanto os níveis remanescentes permaneceram elevados.

As folhas e o fruto do maracujá-amarelo possuem glicosídeos cianogênicos (Medeiros et al., 2009), como a prunasina, sambunigrina e amigdalina (Matsuura et al., 2005). A ingestão destes compostos é tóxica e, como resultado, culmina em distúrbios respiratórios (Medeiros et al., 2009), vertigem, náuseas, vômitos, diarreia e fraqueza (Do Nascimento et al., 2013).

Estudos avaliaram parâmetros de toxicidade após administração com a farinha da casca do fruto maracujá-amarelo (Passiflora edulis f. flavicarpa Deg), entretanto, não obtiveram resultados significativos (Ramos et al., 2007; Medeiros et al., 2009). Estes estudos apresentaram duração máxima de oito semanas, portanto, não é possível afirmar a ausência de efeitos tóxicos com a administração crônica.

Levando em consideração que o processo de trituração não é capaz de atenuar os níveis de glicosídeos cianogênicos presentes na casca do fruto e que os estudos toxicológicos limitaram-se a oito semanas, o emprego crônico desta intervenção com fins de saúde não é comprovadamente seguro.

\section{Agrotóxicos: potencial tóxico e implicações à saúde \\ O termo "agrotóxico" é entendido como substâncias químicas, ou misturas, destinadas}

Rev. Bras. PI. Med., Campinas, v.18, n.2, p.563-569, 2016. 
FIGURA 1. Estudos experimentais envolvendo suplementação com a farinha da casca do maracujá-amarelo (Passiflora edulis f. flavicarpa Deg) e alterações no metabolismo glicídico e lipídico.

\begin{tabular}{|c|c|c|c|c|}
\hline $\begin{array}{l}\text { Autor (es)/ } \\
\text { Ano da } \\
\text { publicação }\end{array}$ & $\mathbf{N}$ & $\begin{array}{l}\text { População } \\
\text { envolvida }\end{array}$ & $\begin{array}{l}\text { Protocolo de } \\
\text { suplementação }\end{array}$ & Resultados \\
\hline $\begin{array}{l}\text { Guertzenstein } \\
\text { \& Sabaa-Srur, } \\
2002\end{array}$ & 36 & $\begin{array}{l}\text { Ratos diabéticos e } \\
\text { não-diabéticos }\end{array}$ & $\begin{array}{l}\text { Administração através da ração } \\
\text { durante } 28 \text { dias. }\end{array}$ & $\begin{array}{l}\text { Animais não-diabéticos tratados } \\
\text { apresentaram redução de } \\
\text { consumo alimentar e peso } \\
\text { corporal, comparado ao grupo } \\
\text { não-diabético placebo. Todos os } \\
\text { grupos tratados apresentaram } \\
\text { redução da glicemia. }\end{array}$ \\
\hline $\begin{array}{l}\text { Braga et al., } \\
2010\end{array}$ & 27 & Ratos diabéticos & $\begin{array}{l}20 \mathrm{mg} / \mathrm{kg}(\mathrm{N}=9) ; 40 \mathrm{mg} / \mathrm{kg}(\mathrm{N}=9) \\
160 \mathrm{mg} / \mathrm{kg}(\mathrm{N}=9) . \text { Os animais } \\
\text { foram suplementados apenas } \\
\text { uma vez durante o experimento. } \\
\text { Administração por gavagem. }\end{array}$ & $\begin{array}{l}\text { Efeito hipoglicemiante em todos } \\
\text { os grupos, entretanto, mais } \\
\text { pronunciado com dose } 160 \mathrm{mg} / \mathrm{kg} \text {. }\end{array}$ \\
\hline $\begin{array}{l}\text { Ramos et al., } \\
2007\end{array}$ & 19 & $\begin{array}{l}\text { Mulheres com } \\
\text { idade entre } 30 \text { e } 60 \\
\text { anos, apresentando } \\
\text { hipercolesterolemia }\end{array}$ & $\begin{array}{l}\text { 30g/dia durante } 60 \text { dias. } \\
\text { Administração via oral, } \\
\text { consumido com as refeições. }\end{array}$ & $\begin{array}{l}\text { Redução nos níveis de colesterol } \\
\text { total e fração LDL. }\end{array}$ \\
\hline $\begin{array}{l}\text { Janebro et al., } \\
2008\end{array}$ & 43 & $\begin{array}{l}\text { Pacientes portadores } \\
\text { de diabetes mellitus } \\
\text { tipo II }\end{array}$ & $\begin{array}{l}30 \mathrm{~g} / \text { dia durante } 60 \text { dias. } \\
\text { Administração via oral, ingerido } \\
\text { com alimentos líquidos. }\end{array}$ & $\begin{array}{l}\text { Redução da glicemia em jejum, } \\
\text { hemoglobina glicada, triglicérides, } \\
\text { aumento de HDL-colesterol. }\end{array}$ \\
\hline $\begin{array}{l}\text { Medeiros et al., } \\
2009\end{array}$ & 36 & $\begin{array}{l}\text { Indivíduos saudáveis } \\
\text { de ambos os sexos }\end{array}$ & $\begin{array}{l}\text { 30g/dia durante oito semanas. } \\
\text { Administração via oral, ingerido } \\
\text { com alimentos líquidos. }\end{array}$ & $\begin{array}{l}\text { Redução da glicemia e peso } \\
\text { corporal. }\end{array}$ \\
\hline $\begin{array}{l}\text { Queiroz et al., } \\
2012\end{array}$ & 43 & $\begin{array}{l}\text { Pacientes portadores } \\
\text { de diabetes mellitus } \\
\text { tipo II }\end{array}$ & $\begin{array}{l}\text { 30g/dia durante } 60 \text { dias. } \\
\text { Administração via oral, } \\
\text { consumido com alimentos. }\end{array}$ & $\begin{array}{l}\text { Redução da glicemia e } \\
\text { hemoglobina glicada }\end{array}$ \\
\hline
\end{tabular}

a prevenir, destruir ou repelir, de forma direta ou indireta, agentes patogênicos, de vida animal ou vegetal, que sejam nocivos às plantas e animais úteis, seus produtos, subprodutos e ao homem (Brasil, 1988).

A contaminação por agrotóxicos pode ocorrer de forma direta ou indireta, através do contato com estas substâncias químicas ou com produtos e ambientes contaminados, respectivamente (Peres et al., 2005). Existem três principais vias de contaminação: ocupacional, ambiental e alimentar (Moreira et al., 2002).

A contaminação por via ocupacional ocorre através da manipulação destes produtos químicos, seja no processo de formulação ou utilização. A via ambiental corresponde à contaminação de águas, atmosfera e solo, enquanto a via alimentar caracteriza-se pela ingestão de produtos contaminados, atingindo, dessa forma, uma vasta parcela da população (Moreira et al., 2002).

Posteriormente a contaminação por agrotóxicos, há manifestação de intoxicação. A intoxicação aguda é decorrente da exposição excessiva aos compostos químicos e se manifesta em poucas horas após a exposição. Os principais sinais e sintomas são fraqueza, náuseas, dores de cabeça, sangramento nasal, convulsões e vômitos. A intoxicação crônica, embora apresente surgimento tardio, culmina em implicações irreversíveis à saúde, como lesões renais e hepáticas, paralisias e neoplasias (Stopelli \& Magalhães, 2005).

Acontaminação crônica por agrotóxicos está 
associada ao desenvolvimento de neurotoxicidade, carcinogênese e alterações no metabolismo endócrino (Stopelli \& Magalhães, 2005). Os canceres de pulmão, próstata, sistema linfático e hematopoiético têm sido os mais citados na literatura (Blair et al., 2015). Referente à atividade hormonal, o hormônio estimulador da tireóide (TSH), após contaminação, é demasiadamente estimulado, favorecendo o quadro de hipotireoidismo. Ademais, a mimetização da atividade estrogênica, através de agrotóxicos, está associada ao desencadeamento do câncer de mama e de testículo (Stopelli \& Magalhães, 2005), bem como a redução da fertilidade (Peres \& Moreira, 2003).

Tendo em vista que o Brasil é considerado, dentre os países em desenvolvimento, o principal mercado consumidor de agrotóxicos (Peres et al., 2007) e que a produtividade do maracujá é prejudicada por doenças e pragas, as quais têm sido combatidas com os defensores agrícolas (Faleiro et al., 2005), o consumo indiscriminado da farinha da casca do fruto maracujá-amarelo, ou seja, sem posologia definida, torna-se preocupante.

\section{CONCLUSÃO}

Os estudos experimentais revisados neste trabalho, de forma unânime, apresentaram eficácia da suplementação com a farinha da casca do fruto maracujá-amarelo (Passiflora edulis f. flavicarpa Deg), referente a ação hipoglicemiante e hipolipidêmica. Este resultado foi observado em estudos com animais e humanos. Não foram constatados efeitos tóxicos nas dosagens e períodos de administração estudados.

A trituração da casca do fruto é incapaz de promover redução de glicosídeos cianogênicos e agrotóxicos, permitindo que os indivíduos submetidos à suplementação estejam susceptíveis aos efeitos tóxicos destes compostos.

Dessa forma, o desenvolvimento de estudos que avaliem a toxicidade da suplementação por períodos crônicos é de suma importância. Referente ao uso de agrotóxicos, a produção da farinha com frutos orgânicos pode ser uma alternativa para atenuar a toxicidade, sendo necessária a elucidação desta hipótese através de estudos.

Vale ressaltar que o domínio de conhecimento pertinente à prescrição e utilização de fitoterápicos deve estar presente no campo de atuação dos profissionais da área da saúde, em especial o nutricionista, visto que este é o profissional mais habilitado na realização de intervenções dietéticas com fins de promoção de saúde, tornando indispensável sua presença.

\section{REFERÊNCIAS}

BELLON, G. et al. Variabilidade genética de acessos silvestres e comerciais de Passiflora edulis Sims. com base em marcadores RAPD1. Revista Brasileira de Fruticultura, v.29, n.1, p. 124-127, 2007.

BENINCÁ, J.P. et al. Evaluation of the anti-inflammatory efficacy of Passiflora edulis. Food Chemistry, v. 104, p. 1097-1105, 2007.

BEZERRA, J.A.F. et al. Extrato de Passiflora edulis na cicatrização de anastomose colônica em ratos: estudo morfológico e tensiométrico. Acta Cirúrgica Brasileira, v. 21 , n. 3, p. 16-25. 2006.

BLAIR, A. et al. Pesticides and human health. Occupational \& Environmental Medicine. v. 72, n.2, p. 81-82, 2015.

BRAGA, A. et al., Investigação da atividade antihiperglicemiante da farinha da casca de Passiflora edulis Sims, Passifloraceae, em ratos diabéticos induzido por aloxano. Revista Brasileira de Farmacognosia. v.20, n.2, p. 186-191, 2010.

BRASIL. Normas Regulamentadoras Rurais $N^{\circ}$ 5 - Produtos químicos. Ministério do Trabalho e Emprego, 12 de abril de 1988. Disponível em: <http:// www.saude.mt.gov.br/cosat/arquivo/1892/normasregulamentadoras> Acesso em: 03 de Jun, 2016.

CARVALHO, F.K.L. et al. Intoxicação experimental por Passiflora foetida (Passifloraceae) em caprinos. Pesquisa Veterinária Brasileira. v.31, n.6, p. 477481, 2011.

CÓRDOVA, K.R. et al. Características físico-químicas da casca do maracujá amarelo (Passiflora edulis Flavicarpa Degener) obtida por secagem. Boletim do Centro de Pesquisa e Processamento de Alimentos ( $B$. CEPPA), v.23, n.2, p. 221-230, 2005.

DENG, J. et al. Anxiolytic and sedative activities of Passiflora edulis f. flavicarpa. Journal of Ethnopharmacology. v.128, p. 148-153, 2010.

DO NASCIMENTO, E.M.G.C. Benefícios e perigos do aproveitamento da casca de maracujá (Passiflora edulis) como ingrediente na produção de alimentos. Revista do Instituto Adolfo Lutz, v. 72, n.3, p. 1-11. 2013.

FERRARI, R. A. et al., Caracterização de subprodutos da industrialização do maracujá aproveitamento das sementes. Revista Brasileira de Fruticultura. v.26, n.1, p. 101-102, 2004.

FREITAS, B.M.; OLIVEIRA FILHO, J.H. Ninhos racionais para mamangava (Xylocopa frontalis) na polinização do maracujá-amarelo (Passiflora edulis). Ciência Rural. v. 33, n. 6, p. 1135-1139, 2003.

GARROS, I.C. et al. Extract from Passiflora edulis on the healing of open wounds in rats: morphometric and histological study. Acta Cirúrgica Brasileira, v. 21, n. 3, p. 55-65. 2006.

GONÇALVES FILHO, A. et al. Effect of Passiflora edulis (passion fruit) extract on rats bladder wound healing: morphological study. Acta Cirúrgica Brasileira, v. 21, n. 2, p. 3-8. 2006.

GUERTZENSTEIN, S.M.J; SABAA SRUR, A.U.O. Uso da casca de maracujá (Passiflora edulis, f. flavicarpa, DEG) cv amarelo na alimentação de ratos (rattus norvergicus) normais e diabéticos. Cadernos do Centro Universitário São Camilo. v.10, p. 213-218, 2002. 
ISHIMOTO, F.Y. et al. Aproveitamento alternativo da casca do maracujá-amarelo (Passiflora edulis f. var. flavicarpa Deg.) para produção de biscoitos. Revista Ciências Exatas e Naturais. v.9, n.2, p. 259-262, 2007.

JANEBRO, D.I. et al. Efeito da farinha da casca do maracujá-amarelo (Passiflora edulis, f.flavicarpa Deg.) nos níveis glicêmicos e lipídicos de pacientes diabéticos tipo 2. Brazilian Journal of Pharmacognosy. v.18, p. 724-732, 2008.

FALEIRO, F.G. et al. Maracujá: germoplasma e melhoramento genético. Edição 1. Planaltina: Embrapa Cerrados, 2005, p. 677.

KONTA, E.M. et al. Evaluation of the antyhipertensive properties of yellow passion fruit pulp (Passiflora edulis Sims f. flavicarpa Deg.) in spontaneously hypertensive rats. Phytotherapy Research. v.28, p. 28-32, 2014.

MARCHI, R. et al. Uso da cor da casca como indicador de qualidade do maracujá amarelo (Passiflora edulis Sims. f. flavicarpaDeg.) destinado à industrialização. Ciência Tecnologia e Alimentos, v. 20, n.3, p. 381-387, 2000.

MATSUURA, F.C.A.U. et al. Effect of grinding and soaking on the reduction of cyanogenic compounds in the albedo of yellow passion fruit. Revista Brasileira de Toxicologia, v. 18, n. 1, p. 63-69, 2005.

MEDEIROS, J.S. et al. Ensaios toxicológicos clínicos da casca do maracujá-amarelo (Passiflora edulis, f.flavicarpa), como alimento com propriedade de saúde. Brazilian Journal of Pharmacognosy. v.19, n.2, p. 394-399, 2009.

MONTANHER, A.B. et al. Evidence of anty-inflammatory effects of Passiflora edulis in na inflammation model. Journal of Ethnopharmacology, v.109, p. 281-288, 2007.

MOREIRA, J.C. et al. Avaliação integrada do impacto do uso de agrotóxicos sobre a saúde humana em uma comunidade agrícola de Nova Friburgo, RJ. Ciência \& Saúde Coletiva. v.7, n.2, p. 299-311, 2002.
OLIVEIRA, L.F. et al. Aproveitamento alternativo da casca do maracujá-amarelo (Passiflora edulis f. flavicarpa) para a produção de doce em calda. Ciência Tecnologia e Alimentos, v.22, n.3, p.259-262, 2002.

PERES, F. et al. Desafios ao estudo da contaminação humana e ambiental por agrotóxicos. Ciência \& Saúde Coletiva. v.10, p. 27-37, 2005.

PERES, F. et al. Os impactos dos agrotóxicos sobre a saúde e o ambiente. Ciência \& Saúde Coletiva. v.12, n.1, p. 4-4, 2007.

PERES, F.; MOREIRA, J.C. É veneno ou é remédio? Rio de Janeiro: Editora Fiocruz, 2003, p.384.

QUEIROZ, M.R.S. et al. Effect of the yellow passion fruit peel flour (Passiflora edulis f. flavicarpa deg.) in insulin sensitivity in type 2 diabetes mellitus patients. Nutrition Journal. v.11, n.89, p. 1-7, 2012.

RAMOS, A.T. et al. Use of Passiflora edulis f. flavicarpa on cholesterol reduction. Revista Brasileira de Farmacognosia, v. 17, n.4, p. 592-597, 2007.

SOUZA, M.M. et al., Cytogenetic studies in some species of Passiflora L. (Passifloraceae): a review emphasizing Brazilian species. Brazilian Archives of Biology and Technology, v.51, n.2, p. 247-258, 2008.

SOUZA, M.S.S. et al. Effects of Passiflora edulis (yellow passion) on serum lipids and oxidative stress status of wistar rats. Journal of Medicinal Food. v.15, n.1, p. 78-82, 2012.

STOPELLI, I.M.B.S.; MAGALHÃES, C.P. Saúde e segurança alimentar: a questão dos agrotóxicos. Ciência \& Saúde Coletiva. v.10, p. 91-100, 2005.

VARGAS, A.J. et al. Passiflora alata and Passiflora edulis spray-dried aqueous extracts inhibit inflammation in mouse modelo of pleurisy. Fitoterapia. v.78, p. 112119, 2007.

ZERAIK, M. L. et al. Maracujá: Um alimento funcional?. Revista Brasileira de farmacognosia, v. 20, n. 3, p. 459-471, 2010. 